\title{
Extended Smoluchowski Model for the Formation of Size-Selected Silver Nanoclusters Generated via Modulated Pulsed Power Magnetron Sputtering
}

Chuhang Zhang, ${ }^{1,2, *}$ Hironori Tsunoyama, ${ }^{2,3}$ Yuanxin Feng, ${ }^{1}$ and Atsushi Nakajima $2,3,4, *$

1) School of Science, Zhejiang University of Science and Technology, Hangzhou 310023, P.R. China

2) JST, ERATO, Nakajima Designer Nanocluster Assembly Project, 3-2-1 Sakado, Takatsu-ku, Kawasaki 213-0012, Japan

3) Department of Chemistry, Faculty of Science and Technology, Keio University, 3-14-1 Hiyoshi, Kohoku-ku, Yokohama 223-8522, Japan

4) Keio Institute of Pure and Applied Science (KiPAS), Keio University, 3-14-1 Hiyoshi, Kohoku-ku, Yokohama 223-8522, Japan 


\section{Supporting Information \\ Details of parameter optimization}

\section{Rough estimation of the initial number density of the sputtered material $\left(n_{0}\right)$}

The estimation of $n_{0}$ was mainly based on the time-resolved mass spectra of Ag nanocluster (NC) anions, as previously reported in the Journal of Physical Chemistry A (see Fig. 5(i)). ${ }^{1}$ The total ion current from the mass spectrum of silver $\mathrm{NC}$ anions, as shown in Fig. 5(i), therein was approximately $17 \mathrm{nA}$. Given that the average size of $\mathrm{NC}$ anions is 40 and the [anion]/[cation] ratio $=1.6$, the initial atom number was $\frac{17 \times 10^{-9} \times 40}{1.6 \times 10^{-19}} \times \frac{2.6}{1.6}=7 \times 10^{12}$ atom $/ \mathrm{s}$. Since the repetition rate was $7 \mathrm{~Hz}$, the initial atom number in each ion bunch was approximately $10^{12}$ atoms. The volume of the ion "bunch" was then estimated. In the experimental setup, the sputtered materials are sufficiently thermalized by collisions with He atoms, which has a lab-frame velocity of $5 \mathrm{~m} / \mathrm{s}$. Therefore, given the time duration of the pulse $(1.5 \mathrm{~ms})$, the length of an ion bunch in the vicinity of the target is approximately $7.5 \times 10^{-3} \mathrm{~m}$. Assuming that the shape of each ion bunch is a hollow cylinder similar to a ring erosion area (inner diameter $=40 \mathrm{~mm}$ and outer diameter $=28$ $\mathrm{mm}$ ), the cross-sectional area is $6 \times 10^{-4} \mathrm{~m}^{2}$, and the volume of each ion bunch in the vicinity of the target is $4.5 \times 10^{-6} \mathrm{~m}^{3}$. Thus, the order of $n_{0}$ for $P_{\mathrm{p}}=320 \mathrm{~W}$ is $10^{18} \mathrm{~m}^{-3}$.

\section{Calculated values for $n_{0}$ and $u$}

According to Ref. 2, the ionization fraction for $P_{\mathrm{p}}=180 \mathrm{~W}$ is $3 \%$. Using this information, the parameter set for $n_{0}$ and $u$, i.e., $\left(n_{0}, u\right)$, was them optimized. Note that at $P_{\mathrm{p}}=180 \mathrm{~W}$, cation-anion aggregation is negligible due to the low material number density. Furthermore, 
based on the above rough estimation, the value for $n_{0}$ at $P_{\mathrm{p}}=180 \mathrm{~W}$ seems to be on the order of $10^{17} \mathrm{~m}^{-3}$. The size distributions with different $(n 0, u)$ are presented in Fig. S1 (a).
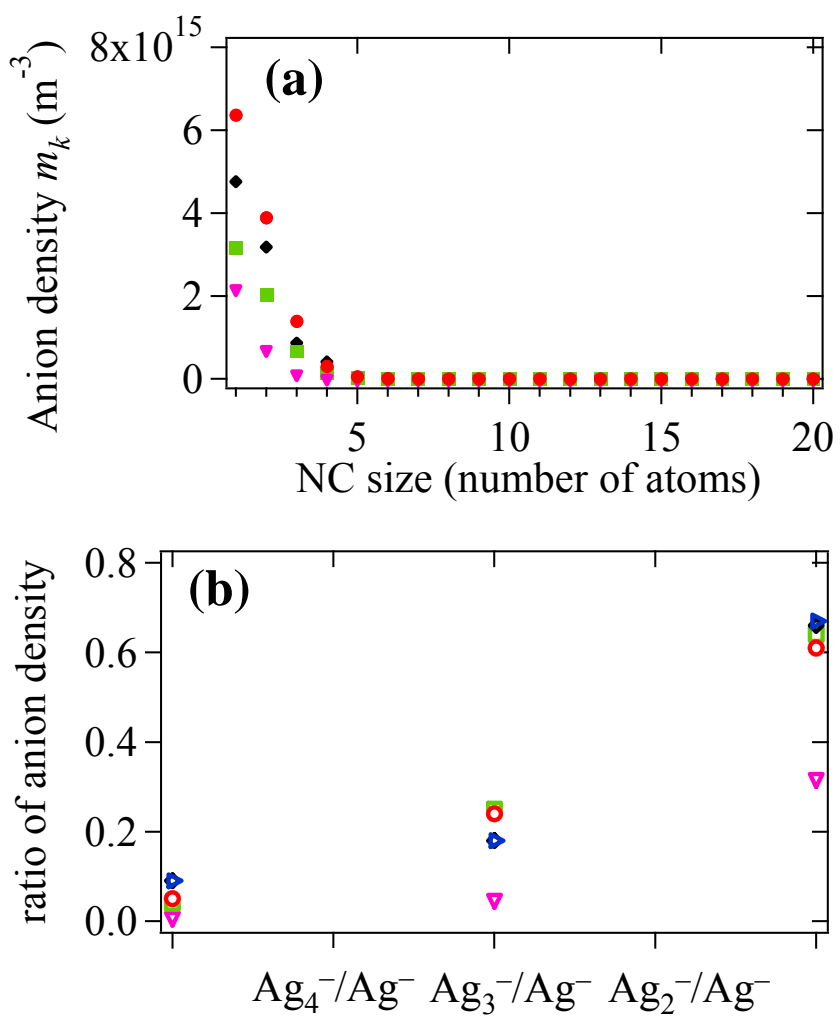

Fig. S1. (a) Size distributions obtained using different parameter sets for $\left(n_{0}, u\right)=\left(10^{17} \mathrm{~m}^{-3}, 6\right)$ (pink), $\left(2 \times 10^{17} \mathrm{~m}^{-3}, 3\right)$ (green), $\left(3 \times 10^{17} \mathrm{~m}^{-3}, 2\right)$ (black), and $\left(4 \times 10^{17} \mathrm{~m}^{-3}, 1.5\right)$ (red), with $\sigma=$ 3\%. (b) Intensity ratios for $\mathrm{Ag}_{4}{ }^{-} / \mathrm{Ag}^{-}, \mathrm{Ag}_{3}{ }^{-} / \mathrm{Ag}^{-}$, and $\mathrm{Ag}_{2}{ }^{-} / \mathrm{Ag}^{-}$from the mass spectrum shown in Fig. 1(a) (blue) and calculated for $(n 0, u)=\left(10^{17} \mathrm{~m}^{-3}, 6\right)$ (pink), $\left(2 \times 10^{17} \mathrm{~m}^{-3}, 3\right)$ (green), $(3 \times$ $\left.10^{17} \mathrm{~m}^{-3}, 2\right)$ (black), and $\left(4 \times 10^{17} \mathrm{~m}^{-3}, 1.5\right)$ (red).

The number density ratios for $\mathrm{Ag}_{4}{ }^{-} / \mathrm{Ag}^{-}, \mathrm{Ag}_{3}{ }^{-} / \mathrm{Ag}^{-}$, and $\mathrm{Ag}_{2}{ }^{-} / \mathrm{Ag}^{-}$were then used to compare the size distributions in Fig. S1 and Fig. 1(a), as shown in Fig. S1 (b). The experimental ratios for $\mathrm{Ag}_{4}{ }^{-} / \mathrm{Ag}^{-}, \mathrm{Ag}_{3}{ }^{-} / \mathrm{Ag}^{-}$, and $\mathrm{Ag}_{2}{ }^{-} / \mathrm{Ag}^{-}$were $0.09,0.18$, and 0.67 , respectively, for the mass spectrum shown in Fig. 1(a). The calculated results obtained for $\left(n_{0}, u\right)=\left(3 \times 10^{17} \mathrm{~m}^{-3}, 2\right)$ well reproduced these ratios $(0.09,018$, and 0.66 , respectively). This good agreement indicates that the parameter set $\left(n_{0}, u\right)=\left(3 \times 10^{17} \mathrm{~m}^{-3}, 2\right)$ is reasonable, as shown in Fig. S1 (b). 


\section{Optimization of the value for " $a$ "}

At high $P_{\mathrm{p}}>300 \mathrm{~W}, \mathrm{NC}$ cation-anion aggregation cannot be ignored. The attractive potential between the cations and anions can be taken into account by fitting the size distributions using the rate constant $K_{i, j}^{\prime \prime}=a K_{i, j}$, where " $a$ " is a constant. The calculated size distributions were compared to those observed in the corresponding mass spectra, and the optimized value for $a$ was determined by varying the values $\left(n_{0}, u\right)$. Because, according to Refs. 2 and 3 , the values for $n_{0}$ and $\sigma$ increase linearly with $P_{\mathrm{p}}, n_{0}=10^{18} \mathrm{~m}^{-3}$ was used for $P_{\mathrm{p}}=500 \mathrm{~W}$. On the other hand, $u=2$ was used because it is approximately independent of $P_{\mathrm{p}}$.
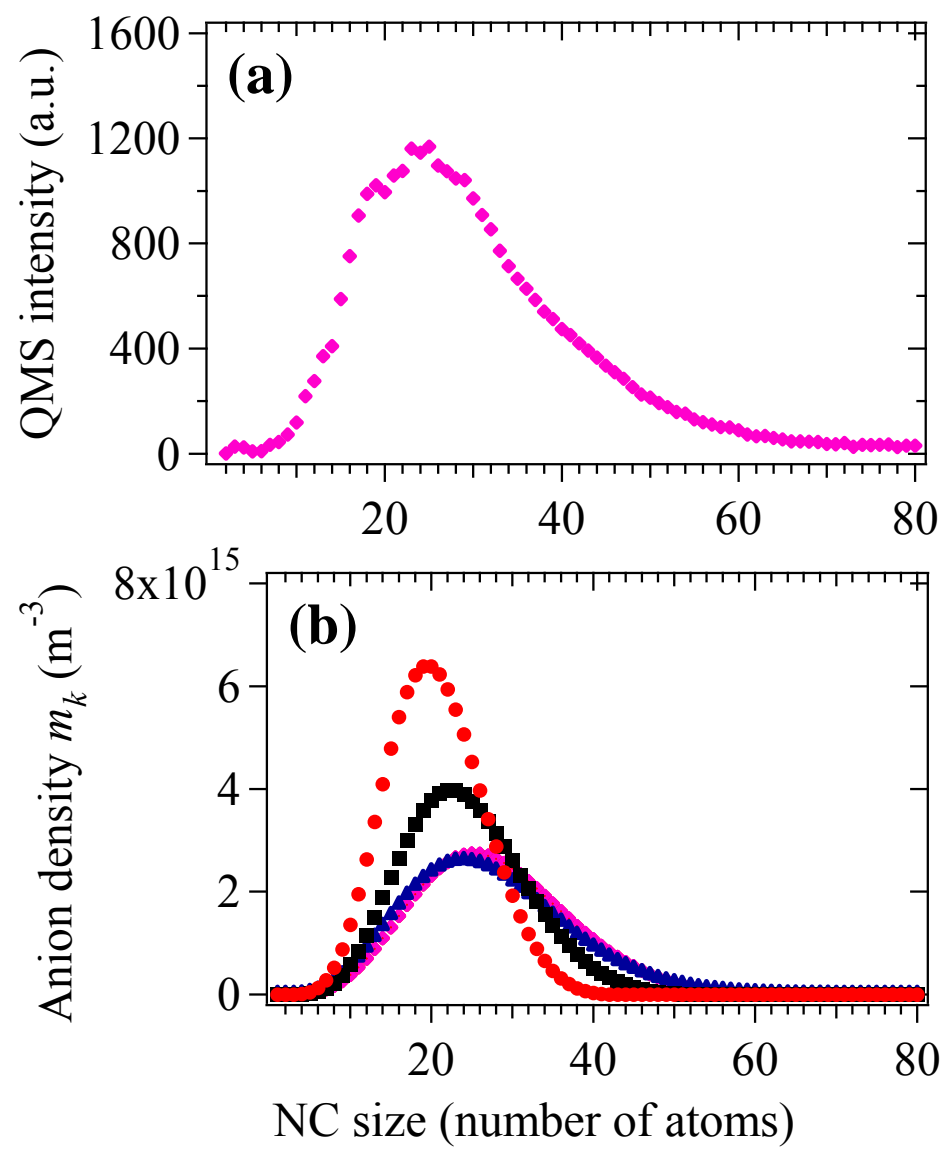

Fig. S2. Optimization of the coefficient " $a$ " of the rate constant for interactions between NC cations and anions. (a) Mass spectrum of silver NC anions generated at $P_{\mathrm{p}}=500 \mathrm{~W}$ and $f=10 \mathrm{~Hz}$. (b) Calculated size distributions at $a=3$ (blue), 4 (pink), 5 (black), and 9 (red). Other parameters: $n_{0}=10^{18} \mathrm{~m}^{-3}, \sigma=11 \%$, and $u=2$. 
The mass spectrum of $\mathrm{Ag} \mathrm{NC}$ anions generated at $P_{\mathrm{p}}=500 \mathrm{~W}$ and the simulated results obtained using different values for $a$ are presented in Figs. S2 (a) and (b), respectively. The NC size with the greatest peak intensity in the mass spectrum was the 26-mer, which was consistently simulated when $a=4$. Furthermore, the size distributions were also reproduced when $a=4$. Therefore, the optimized coefficient of the rate constant for interactions between NC cations and anions was determined to be $a=4$.

\section{Mass spectra for NC ions, their corresponding NC neutrals, and their calculated size distributions}

The size distributions of $\mathrm{NC}$ anions, cations, and neutrals were examined under the same sputtering conditions. To measure their corresponding $\mathrm{NC}$ neutrals, a higher repetition rate of $f=$ $130 \mathrm{~Hz}$ was used because the neutrals were detected via electron-impact ionization. Figures S3 and S4 show the dependence of the size distributions of the NC ions and neutrals, respectively, at peak power $\left(P_{\mathrm{p}}\right)$. As $P_{\mathrm{p}}$ increased from $200 \mathrm{~W}$ to $800 \mathrm{~W}$, the average size of the $\mathrm{NC}$ anions gradually shifted from approximately 20 -mers to 45 -mers. Simultaneously, with increasing $P_{\mathrm{p}}$, the population of larger NC cations (larger than 10-mers) gradually increased to $800 \mathrm{~W}$, as shown in Fig. S3(b). For the neutrals, NCs with sizes of 30-mers to 130 -mers appeared above $P_{\mathrm{p}}=400$ W and with increasing $P_{\mathrm{p}}$, the average size gradually increased to $800 \mathrm{~W}$. Further increases in $P_{\mathrm{p}}$ from $800 \mathrm{~W}$ to $1,000 \mathrm{~W}$ caused a reduction in not only the NC size and number density of NC anions and cations but also the number density of the NC neutrals. 

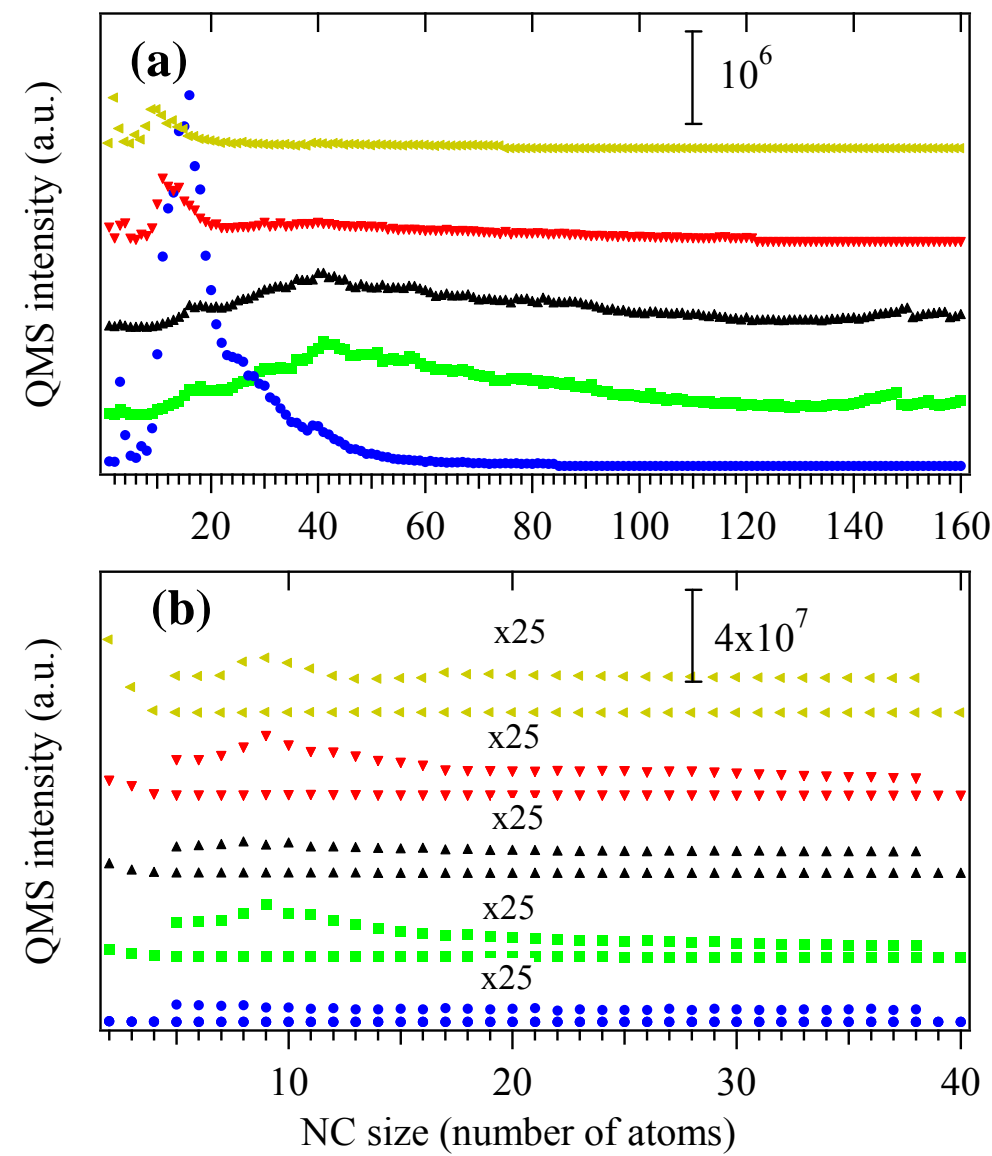

Fig. S3. Mass spectra of NC (a) anions and (b) cations generated by high-power impulse magnetron sputtering (HiPIMS) with varied $P_{\mathrm{p}}$ : $200 \mathrm{~W}$ (blue), $400 \mathrm{~W}$ (green), $600 \mathrm{~W}$ (black), 800 $\mathrm{W}$ (red), and $1000 \mathrm{~W}$ (yellow). Repetition rate: $130 \mathrm{~Hz}$, He flow rate: $300 \mathrm{sccm}$, Ar flow rate: 90 $\mathrm{sccm}$. The scale bar indicates the intensity of the NCs in counts per second.

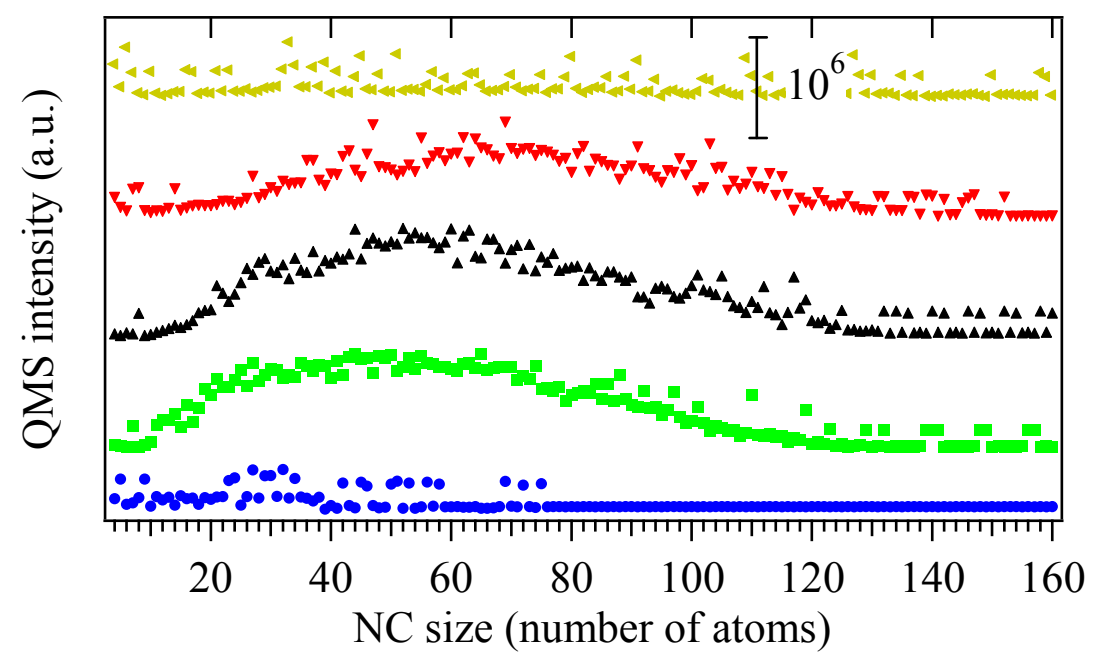

Fig. S4. Mass spectra of NC neutrals generated by HiPIMS with varied $P_{\mathrm{p}}$ : $200 \mathrm{~W}$ (blue), $400 \mathrm{~W}$ (green), $600 \mathrm{~W}$ (black), $800 \mathrm{~W}$ (red), and $1000 \mathrm{~W}$ (yellow). Repetition rate: $130 \mathrm{~Hz}$, He flow rate: $300 \mathrm{sccm}$, Ar flow rate: $90 \mathrm{sccm}$. The scale bar indicates the intensity of the NCs in counts per second. 
The size distributions of the NC anions and cations simulated using the extended Smoluchowski model with all parameters adjusted for $f=130 \mathrm{~Hz}$ are presented in Fig. S5 for comparison. It is found that the sizes of the $\mathrm{NC}$ cations are usually smaller than those of the NC anions owing to the lower initial number density of atomic cations (atomic anion/cation $=1.6$ ). The size of the NC cations increases with $P_{\mathrm{p}}$; this is similar to the results obtained in the mass spectra (Fig. S3(b)). Even though the degree of size-shift with increasing $P_{\mathrm{p}}$ is relatively larger for $\mathrm{NC}$ anions than for $\mathrm{NC}$ cations (Fig. S5(a)), the trend in the size-shift was qualitatively reproduced in the simulations.
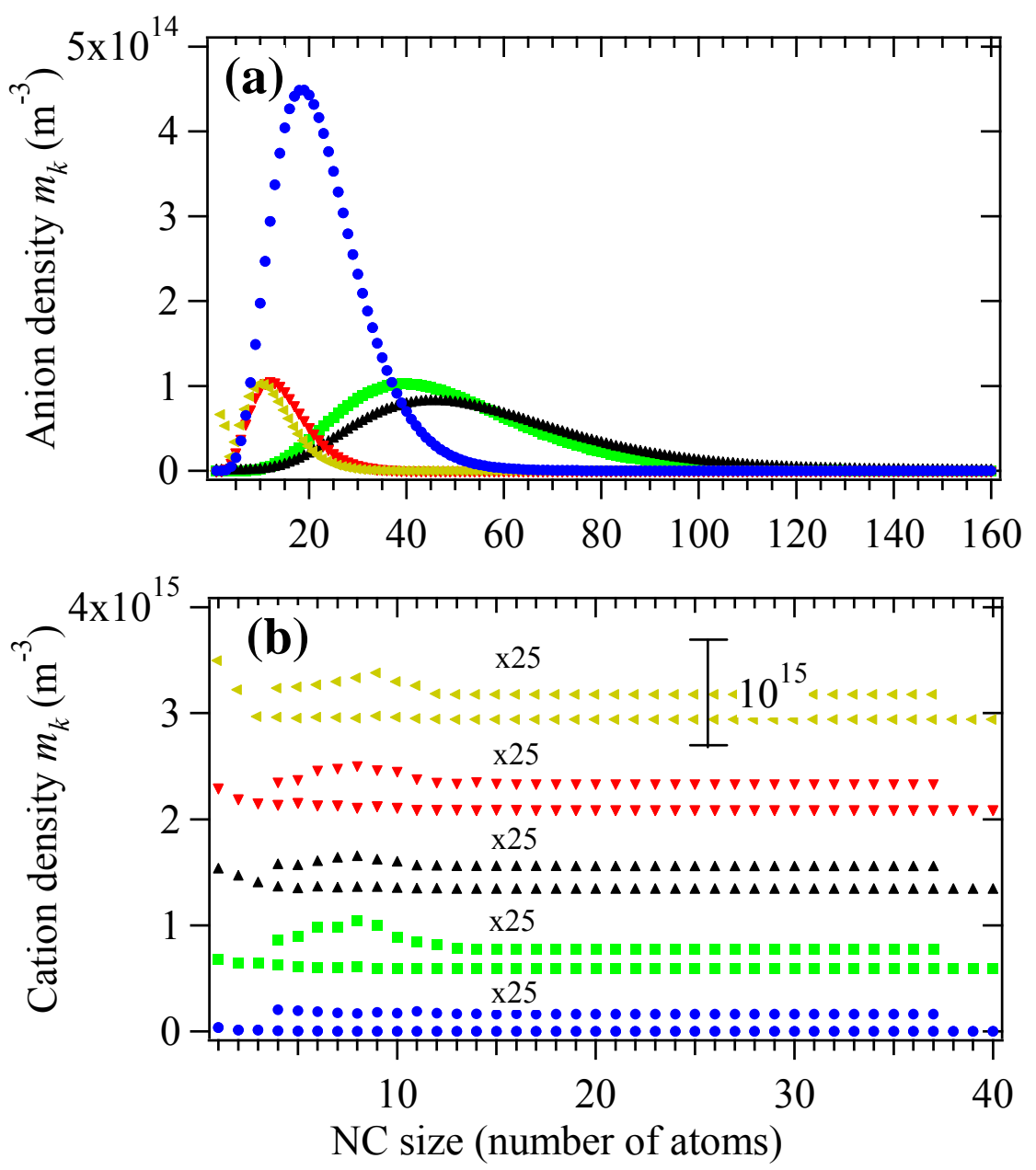

Fig. S5. Calculated size distributions of $\mathrm{NC}(\mathrm{a})$ anions and (b) cations for $\left(n_{0}, \sigma\right)=\left(5.0 \times 10^{18} \mathrm{~m}^{-3}\right.$, $4 \%)$ (blue), $\left(8.0 \times 10^{18} \mathrm{~m}^{-3}, 9 \%\right)$ (green), $\left(1.2 \times 10^{19} \mathrm{~m}^{-3}, 12 \%\right)$ (black), $\left(1.8 \times 10^{19} \mathrm{~m}^{-3}, 18 \%\right)$ (red), and $\left(2.0 \times 10^{19} \mathrm{~m}^{-3}, 20 \%\right)$ (yellow). The scale bar indicates the density of the NCs in $\mathrm{m}^{-3}$. 
To further investigate the underlying physics of the size reduction in NC ions, the size distributions of the NC neutrals were also studied. Figures S4 and S6 show experimental and simulated size distributions of $\mathrm{NC}$ neutrals under varied $\left(n_{0}, \sigma\right)$, corresponding to $P_{\mathrm{p}}=200 \mathrm{~W}$, $400 \mathrm{~W}, 600 \mathrm{~W}, 800 \mathrm{~W}$, and $1000 \mathrm{~W}$. The size distributions of the corresponding NC neutrals $\left(\mathrm{Ag}_{n}\right)$ are significantly wide compared with those of the NC ions because of the relatively high initial number density of $\mathrm{Ag}$ atoms at low peak power $P_{\mathrm{p}}$. The most populated size-shift of the NC neutrals is from approximately 50 -mers to 65 -mers as $P_{\mathrm{p}}$ increases from $400 \mathrm{~W}$ to $800 \mathrm{~W}$ (Fig. S6); this is consistent with the results observed in the mass spectra (Fig. S4). Meanwhile, the simulated size distributions are also quite similar to those observed in the experimental mass spectra. The dependence of the NC sizes for cations, anions, and neutrals on $P_{\mathrm{p}}$ at $f=130 \mathrm{~Hz}$ is qualitatively summarized as follows: (1) for $P_{\mathrm{p}}<400 \mathrm{~W}$, the sizes of all three types of NCs gradually increase with $P_{\mathrm{p}}$; (2) in the power range $400 \mathrm{~W}<P_{\mathrm{p}}<600 \mathrm{~W}$, the size remains unchanged for the NC cations and anions, while the NC neutrals gradually become larger; (3) in the power range $600 \mathrm{~W}<P_{\mathrm{p}}<800 \mathrm{~W}$, the size of cations and anions begin to decrease with increasing $P_{\mathrm{p}}$ in contrast to the further increase in size for the neutrals; and (4) for $800 \mathrm{~W}<P_{\mathrm{p}}$, the sizes of the NC cations and anions further decrease and the intensity of the neutral NCs drops dramatically with increasing $P_{\mathrm{p}}$.

The agreement between the simulations and experiments in the power range $500 \mathrm{~W}<P_{\mathrm{p}}<$ $800 \mathrm{~W}$ suggests that the origin of the size reduction in $\mathrm{NC}$ ions is primarily because of an accelerated NC anion-cation aggregation. Since the dramatic reduction in intensity cannot be explained by the extended Smoluchowski model, the reason for the reduction in size for the NC ions above $P_{\mathrm{p}}=800 \mathrm{~W}$ seemingly originates from a hindrance to the NC growth owing to the 
elevated temperature of the buffer gas rather than the aggregation of NC anions-cations, which is increases because of the high plasma temperature for $P_{\mathrm{p}}>800 \mathrm{~W}$.

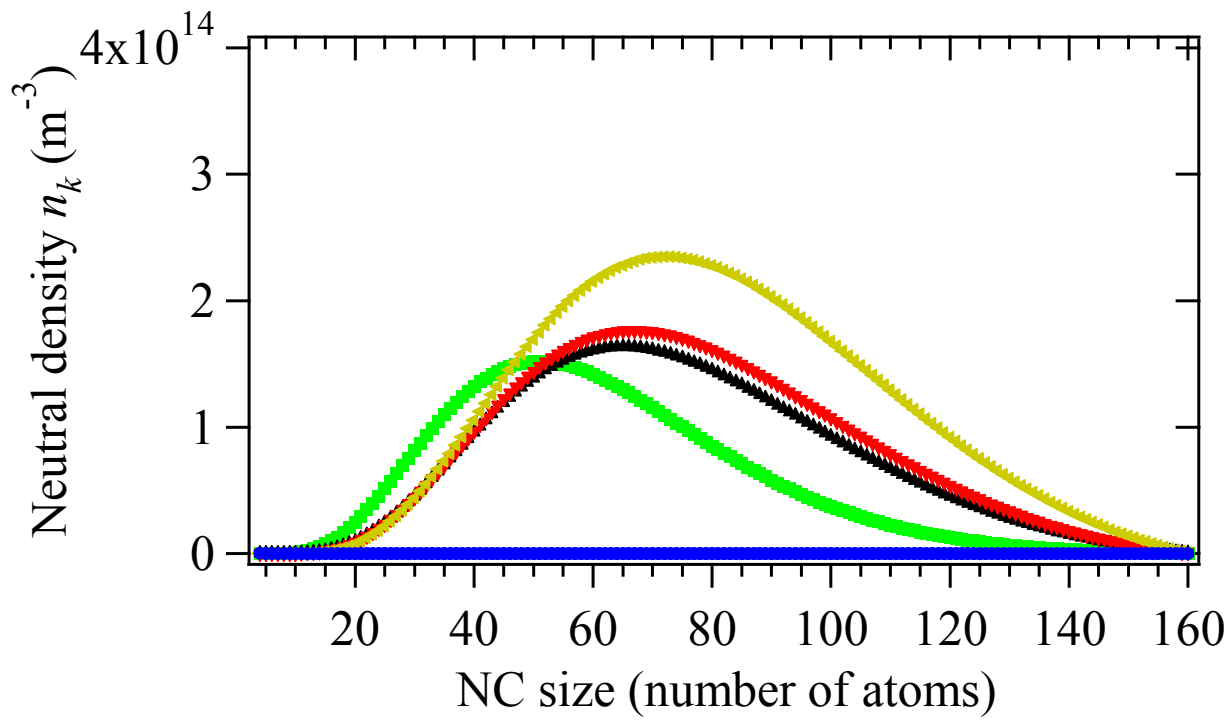

Fig. S6. Calculated size distributions of the NC neutrals for $\left(n_{0}, \sigma\right)=\left(5.0 \times 10^{18} \mathrm{~m}^{-3}, 4 \%\right)$ (blue), $\left(8.0 \times 10^{18} \mathrm{~m}^{-3}, 9 \%\right)($ green $),\left(1.2 \times 10^{19} \mathrm{~m}^{-3}, 12 \%\right)$ (black), $\left(1.8 \times 10^{19} \mathrm{~m}^{-3}, 18 \%\right)$ (red), and $(2.0$ $\times 10^{19} \mathrm{~m}^{-3}, 20 \%$ ) (yellow).

\section{References}

1) Zhang, C.H.; Tsunoyama, H.; Akatsuka, H.; Sekiya, H.; Nagase, T.; Nakajima, A. Advanced nanocluster ion source based on high-power impulse magnetron sputtering and time-resolved measurements of nanocluster formation. J. Phys. Chem. A 2013, 117, 10211-10217.

2) Hala, M.; Capek, J.; Zabeida, J.; Klemberg-Sapieha, J.E.; Martinu, L. Pulse management in high power pulsed magnetron sputtering of niobium. Surf. Coat. Technol. 2012, 206, 4186-4193.

3) Helmersson, U.; Lattemann, M.; Bohlmark, J.; Ehiasarian, A.P.; Gudmundsson, J.T. Ionized physical vapor deposition (IPVD): A review of technology and applications. Thin Solid Films 2006, 513, 1-24. 